\title{
Photoisomerization for a molecular switch in contact with a surface
}

\author{
Jörg Henzl, ${ }^{1}$ Peter Puschnig, ${ }^{2}$ Claudia Ambrosch-Draxl, ${ }^{2}$ Andreas Schaate, ${ }^{3}$ Boris Ufer, ${ }^{3}$ Peter Behrens,${ }^{3}$ and \\ Karina Morgenstern ${ }^{3, *}$ \\ ${ }^{1}$ Institut für Festkörperphysik, Abteilung ATMOS, Leibniz Universität Hannover, Appelstrasse 2, D-30167 Hannover, Germany \\ ${ }^{2}$ Lehrstuhl für Atomistic Modelling and Design of Materials, Montanuniversität Leoben, Franz-Josef-Strasse 18, A-8700 Leoben, Austria \\ ${ }^{3}$ Institut für Anorganische Chemie, Leibniz Universität Hannover, Callinstrasse 9, D-30167 Hannover, Germany
}

(Received 9 December 2011; published 6 January 2012)

\begin{abstract}
The photoisomerization properties of an azobenzene derivative on a thin insulating $\mathrm{NaCl}$ layer on $\mathrm{Ag}(111)$ are investigated with a low-temperature scanning tunneling microscope and density functional calculations. Illumination with UV light at $365 \mathrm{~nm}$ induces the reversible direct isomerization of the adsorbed species, while visible light does not lead to any changes. This unexpected behavior cannot be explained by the change of the electronic structure upon adsorption on the inert surface. It is rationalized in terms of electrostatic interactions caused by the atomistic details of the surface.
\end{abstract}

DOI: 10.1103/PhysRevB.85.035410

PACS number(s): 68.37.Ef, 68.43.Hn, 68.65.-k, 82.37.Gk

\section{INTRODUCTION}

Future nanoelectronics and nanomachines demand active elements on a molecular scale to trigger and to control their functionality. ${ }^{1}$ Photochromic molecules are highly attractive candidates for this purpose because light can reversibly switch them between two different states. ${ }^{2}$ Among photochromic molecules, azobenzene derivatives are extensively studied because of their robust photoisomerization in solution phase ${ }^{2}$ and on top of self-assembled monolayers. ${ }^{3}$ For a potential implementation into real devices, however, the active elements, including the photochromic molecules, have to work under different environments, especially in contact with a surface. This contact with a surface is expected to alter the photoisomerization properties substantially, e.g., by bond formation, steric hindrance, or electronic quenching. Indeed, the photoisomerization of native azobenzene molecules directly adsorbed on a metal substrate is suppressed ${ }^{4}$ presumably because of a shorter lifetime of the photoexcited state on the metal surface. As a consequence, azobenzene molecules have been functionalized with tert-butyl spacer groups, which are intended to lift the azobenzene scaffold away from the surface, while keeping the molecular plane parallel to it. Although exposure to light led to isomerization of the azobenzene derivative on $\mathrm{Au}(111),{ }^{4-6}$ this was not related to the direct photoisomerization of the molecule. Rather, the light was first absorbed by the $d$-band of the metal and then the isomerization was induced by an electron transfer from the molecule to the thus created hole in the $d$-bands. ${ }^{5}$

Another attempt for decoupling of a dye molecule from a $\mathrm{Cu}(111)$ surface was based on physisorption. ${ }^{7}$ In this study, the molecules could be switched between the isomers by exposure to UV light, while usually on this surface the isomerization is completely quenched. The position of the $d$-bands of copper suggested that the substrate-mediated process is not accessible.

Here, we report the direct photoisomerization of a bistable azobenzene molecule, 4,4'-dihydroxyazobenzene [DHA, see Figs. 1(a) and 1(b)], in contact with a surface using a mercury arc lamp. We achieve this by decoupling the molecule from the metal substrate by a thin insulating layer of sodium chloride $(\mathrm{NaCl}) .{ }^{8-10}$ We investigate the two isomer configurations and the isomerization process by STM at $5 \mathrm{~K}$. By wavelength- dependent measurements, we reveal that photoisomerization is possible via one out of two possible pathways only. In the liquid phase, the rotation pathway follows excitation of the $\pi-\pi^{*}$ transition [cf. Fig. 2(h), inset, below] by UV light and the inversion pathway follows the excitation of the $n-\pi^{*}$ transition by visible light. ${ }^{11}$ On the $\mathrm{NaCl}$ surface, the latter pathway is suppressed. Possible origins of this quenching are discussed based on density functional calculations.

\section{EXPERIMENTAL AND THEORETICAL METHODS}

The measurements are performed with a scanning tunneling microscope at $5 \mathrm{~K}$ under UHV conditions. The 4,4'dihydroxyazobenzene molecules are deposited at $17 \mathrm{~K}$ onto $\mathrm{NaCl}$ islands grown on a clean $\mathrm{Ag}(111)$ surface. The sample is illuminated in situ the STM by a $100 \mathrm{~W}$ mercury lamp with different filters. The angle of incident is $85^{\circ}$ and the spot size is approximately $1.1 \mathrm{~cm}^{2}$. The intensities on the sample are $80 \mathrm{~mW} \mathrm{~cm}{ }^{-2}$ for the UV filter and $442 \mathrm{~mW} \mathrm{~cm}^{-2}$ for the vis filter. During illumination, the STM tip is retracted as far as possible away from the sample and the incoming light (up to $250 \mathrm{~nm}$ vertically and up to $450 \mathrm{~nm}$ horizontally) in order to minimize the shadowing effect of the tip. The absorption spectra in 1,4-dioxane were determined by means of a Cary 5E UV-vis-NIR spectrophotometer using a quartz cuvette with the solvent as the reference standard. The irradiation of the solution is done by an $8 \mathrm{~W}$ laboratory UV lamp of type NU-8 $\mathrm{KL}$ with an emission maximum at $366 \mathrm{~nm}$.

Adsorption geometry and electronic structure of both DHA isomers are calculated within density functional theory. Our structural model is based on a (4 0,2 6) overlayer structure of $\mathrm{NaCl}$ on an $\mathrm{Ag}(111)$ surface with the lattice vectors $\mathbf{a}=$ $(1.157,0,0) \mathrm{nm}$ and $\mathbf{b}=(1.446,1.503,0) \mathrm{nm}$. With an experimental lattice parameter of $a_{\mathrm{Ag}}=0.409 \mathrm{~nm}$ for silver and a bulk lattice constant of $a_{\mathrm{NaCl}}=0.564 \mathrm{~nm}$, this results in $2.5 \%$ and $4.5 \%$ strains in the $\mathrm{NaCl}$ layers along the two unit-cell vectors, respectively. We use an asymmetric repeated slab approach with three layers of $\mathrm{Ag}$, two $\mathrm{NaCl}$ layers, and the DHA molecule adsorbed on the top side. For the determination of the adsorption geometry, we allow all structural degrees of freedom to be relaxed except for the positions of the bottom two Ag layers. We treat exchange-correlation effects 

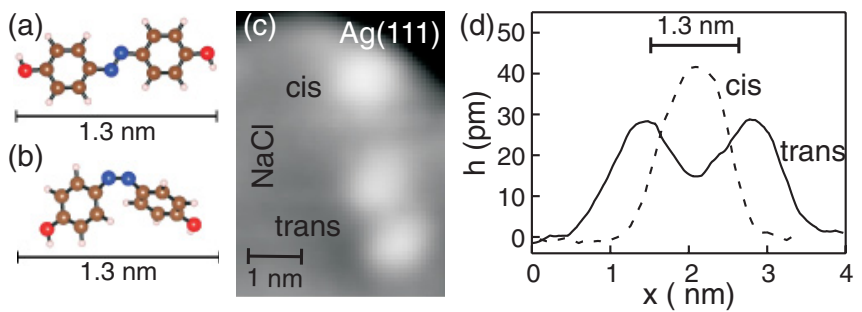

FIG. 1. (Color online) 4,4'-dihydroxyazobenzene molecules on a $\mathrm{NaCl}$ double layer on $\mathrm{Ag}(111)$. (a), (b) Ball-and-stick models of (a) the trans- and (b) the cis-isomer as optimized in gas phase. (c) STM image of a trans- and a cis-isomer. The black area on top of the image is the $\mathrm{Ag}(111)$ surface, the gray area is the $\mathrm{NaCl}$ surface, and the white protrusions are molecules. $I=30 \mathrm{pA}, V=306 \mathrm{mV}$. (d) Line scans across a trans- (solid line) and a cis-isomer (dashed line) similar to the ones shown in (c) and measured across the highest points. The apparent heights of the trans-configuration and cis-configuration are only $(29 \pm 5)$ and $(41 \pm 5)$ pm, respectively.

by the generalized gradient approximation ${ }^{12}$ and take into account van der Waals interactions by an empirical correction scheme. ${ }^{13}$ We use the VASP code with the projector augmented wave method, ${ }^{14}$ a plane-wave cutoff of $285 \mathrm{eV}$, and a $k$ grid of $3 \times 2 \times 1$.

\section{RESULTS AND DISCUSSION}

Scanning tunneling microscopy (STM) investigations have proven the principal feasibility of isomerization of azobenzene derivatives on metal surfaces by electrons or by the electric field of the STM tip and have established the appearance of this molecule in STM images. ${ }^{15-17}$ The trans-configuration of other azobenzene derivatives on metal surfaces is imaged by STM as a double protrusion with a distance between the protrusions in the region of $0.5-1.5 \mathrm{~nm} \cdot{ }^{15-17}$ Indeed, double protrusions with a distance of $(1.31 \pm 0.08) \mathrm{nm}$ are observed after the deposition of DHA on $\mathrm{NaCl}$ [see Fig. 1(c) and the line scan in Fig. 1(d)]. This length fits to a planar adsorbed molecule with the end groups being the most prominent features in the STM images [see the ball-and-stick model in Fig. 1(a)] as expected for a physisorbed molecule. ${ }^{18}$

We illuminate such trans-isomers with UV light $(365 \mathrm{~nm})$ for up to $18 \mathrm{~h}$. The large-scale images in Figs. 2(a) and 2(b) demonstrate that we are able to image the same area of the surface after this illumination. The trans-isomer is converted into a single protrusion [Figs. 2(c) and 2(d) $]^{19}$ with a slightly asymmetric shape and a larger apparent height as compared to the double protrusion. A line scan of a similar protrusion as found directly after adsorption is shown in Fig. 1(d). Qualitatively similar ellipsoidal protrusions were identified before as the cis-configuration of unsubstituted azobenzene molecules on $\mathrm{Au}(111)$ (Ref. 16) and of amino-nitro-azobenzene on $\mathrm{NaCl} / \mathrm{Ag}(111) .{ }^{20}$ Note that the interpretation of the STM images is in line with a multitude of previous studies of azobenzene derivatives. ${ }^{21}$

The cis-configuration can be converted back into the transconfiguration by illumination with UV light $(365 \mathrm{~nm})$, which is evidenced by the STM images in Figs. 2(e) and 2(f) taken before and after light exposure, respectively.
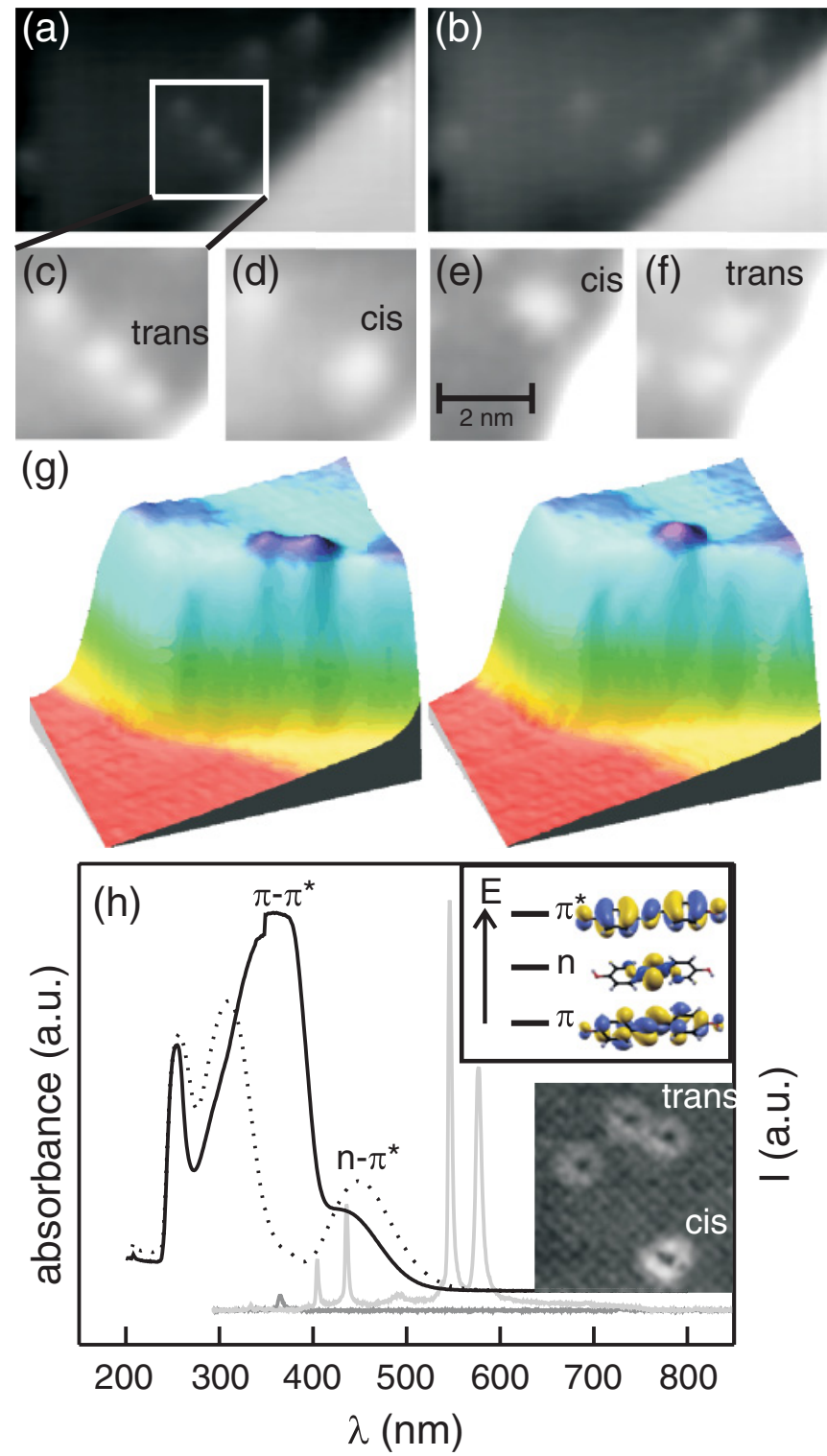

FIG. 2. (Color online) Photoisomerization of 4,4'dihydroxyazobenzene on $\mathrm{NaCl}$. (a) and (b) Large-scale images showing diffusion of molecular parts and isomerization as magnified in (c) and (d) Trans-cis isomerization with enhanced contrast. (e) and (f) Cis-trans isomerization from different experiment. (a), (c), (e) Before and (b), (d), (f) after light exposure for $18 \mathrm{~h}$. Surface areas on the bottom right are higher because of a buried Ag step beneath the $\mathrm{NaCl}$ layer. $I=26 \mathrm{pA}, V=196 \mathrm{mV}$. Note that images $(\mathrm{a}, \mathrm{b})$ are cut outs of even larger images and that the $\mathrm{NaCl}$ island terminates on the left-hand side. (g) 3D false color image of another example close to the $\mathrm{NaCl}$ edge; $\mathrm{Ag}(111)$ is colored red, $\mathrm{NaCl}$ bilayer yellow to blue, and isomers purple. (h) Absorption spectrum of trans- (black line) and cis- (black dotted line) dihydroxyazobenzene in 1,4-dioxane and spectrum of $\mathrm{Hg}$ lamp with different filters. Gray: UV filter. Light gray: vis filter. Upper inset shows corresponding orbitals for native azobenzene. Lower inset shows simultaneous atomic resolution of chlorine lattice imaged with functionalized tip, 28 pA, $193 \mathrm{mV}$.

The long exposure times in connection with the thermal drift induced by the temperature increase during illumination did not allow us to observe the reversibility of the process 

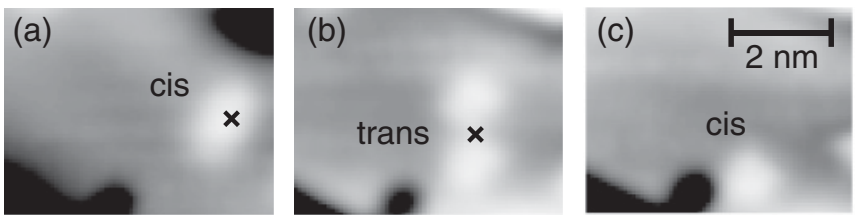

FIG. 3. Electron-induced isomerization: Cross indicates tip position during manipulation with $-800 \mathrm{mV}, 100 \mathrm{pA}$ for $2 \mathrm{~s}$. (a) and (b) Cis to trans isomerization. (b) and (c) Trans to cis isomerization. $I=30 \mathrm{pA}, V=306 \mathrm{mV}$.

at the same molecule. ${ }^{22}$ We thus demonstrate reversibility based on manipulation experiments with the STM (Fig. 3). These manipulations trigger the reversible isomerization by inelastically tunneling electrons (cf. Ref. 20). Both, trans-cis and cis-trans isomerization can be induced with the same bias voltages above $0.8 \mathrm{~V}$ in both polarities. A threshold in this region corresponds to the height of the energy barrier in the ground state between the two isomers. ${ }^{21}$

We continue by discussing possible mechanisms of the UVlight-induced isomerization demonstrated in Fig. 2 concluding that it is triggered via direct light absorption by the molecule. Thermal isomerization is excluded because the temperature during light exposure rises by less than $5 \mathrm{~K}$. The corresponding thermal energy at $10 \mathrm{~K}$ is far below the ground-state isomerization barrier of $\approx 0.8 \mathrm{~V}^{23}$ Furthermore, annealing the sample at $10 \mathrm{~K}$ for the longest illumination time of $18 \mathrm{~h}$ does not lead to any changes. Indirect, i.e., substrate-mediated, isomerization is unlikely due to the presence of the $0.56-\mathrm{nm}$ thick $\mathrm{NaCl}$ spacer layer, which reduces the transfer probability of the photoexcited substrate electrons to a negligible value. Furthermore, the energy of the UV light (365 nm, $3.4 \mathrm{eV})$ is not sufficient to excite $\operatorname{Ag}(111) d$-band electrons (band edge $\approx 4 \mathrm{eV}$ below the Fermi energy), which triggered the above-discussed isomerization of tetra-tert-butyl-azobenzene on $\mathrm{Au}(111){ }^{5}$

The direct photoexcitation at $365 \mathrm{~nm}$ leads to isomerization of approximately $40 \%$ and $71 \%$ of the trans-molecules after 13 and $15 \mathrm{~h}$, respectively. After $18 \mathrm{~h}$, corresponding to a photon dose of $1.4 \times 10^{22} \mathrm{~cm}^{-2}$, nearly all trans-isomers are converted into cis-isomers $(82 \pm 18) \%$. The statistics is based on roughly 50 molecules. The stationary state is thus reached after only a five-times-higher photon dose than for the substrate-mediated photoisomerization of tetra-tert-butylazobenzene on $\mathrm{Au}(111)\left[\approx 3 \times 10^{21} \mathrm{~cm}^{2}\right.$ (Ref. 24)], for which a 70:30 ratio could be reached.

In order to further elucidate the isomerization process, we analyze its wavelength dependence. First, the wavelength of the UV filter $(365 \mathrm{~nm})$ is compared to the absorbance spectra of the DHA molecules in 1,4-dioxane [Fig. 2(h)]. It matches the $\pi-\pi^{*}$ absorption peak of the trans-configuration. This transition is followed by the so-called rotation pathway, in which the trans-to-cis isomerization is achieved by rotating the molecule around the $\mathrm{N}-\mathrm{N}$ double bond.

The probability for the reversed switching is only about $5 \%$ within $18 \mathrm{~h}$ in our experiment. The smaller probability of cistrans isomerization as compared to trans-cis isomerization is in agreement with the reduced intensity of the $\pi-\pi^{*}$ adsorption peak for the cis-isomer [Fig. 2(g)].
On the other hand, it is known from liquid phase experiments that exciting the $n-\pi^{*}$ transition by visible light results in the inversion pathway. ${ }^{2}$ In this case, the cis-trans isomerization is accomplished by an inversion of the $\mathrm{N}-\mathrm{C}$ bond with the nitrogen's lone pair orbital. We thus illuminate the sample with the vis filtered light. Although we use a three-times-larger photon dose of $5 \times 10^{22} \mathrm{~cm}^{-2}$, no isomerization is observed for the adsorbed DHA. From our experiments, we conclude that the rotation pathway is active also for the adsorbed molecule while the inversion pathway is efficiently suppressed by adsorption of the molecule onto the $\mathrm{NaCl}$.

We now discuss possible reasons for this suppression of the inversion pathway. Adsorption onto the surface could affect three processes. First, the switching of a dye molecule is initiated by absorbance of the light. Second, the excitation should live long enough to allow for the much slower change in nuclear positions. Finally, the motion of the different molecular parts should not be hindered. To identify the affected process, we calculate the electronic structure of adsorbed DHA by DFT and compare it to that of the isolated molecule. The adsorption site used as a starting point in the calculations is supported from experiment by employing a functionalized tip, ${ }^{20}$ which allows us to image the molecules (though with a different shape) and the chlorine lattice simultaneously [lower inset in Fig. 2(g)]. Though it is not obvious how the molecule is imaged, we utilize the symmetry of the molecule to identify the adsorption tip. The symmetry implies that the azo-group is positioned in the middle between these two circles. The common motif of all adsorption sites is the position of the azo-group above a fourfold hollow site, in between two chlorine and two sodium atoms similar to the adsorption of amino-nitro-azobenzene on the same surface. ${ }^{20}$ As the understanding of imaging with functionalized tips is still at its infancy, these determinations are not beyond all doubt. However, the calculation showed that the position of the azo-group is of minor importance as binding is through the hydroxyl end groups.

Figures 4(a) and 4(b) display top and side views of the relaxed trans-DHA adsorption geometry, respectively, while Figs. 4(d) and 4(e) show the corresponding cis-adsorption geometry. We rationalize the computed adsorption sites and geometries in terms of electrostatic interactions between the hydroxy end groups and the ionic $\mathrm{NaCl}$ surface. For the transisomer, the negatively charged oxygen atoms are pulled toward the $\mathrm{Na}$ cations leading to an overall bending of the molecule with an O-Na distance of $0.28 \mathrm{~nm}$ while the azo-group is $50 \mathrm{pm}$ higher [Fig. 4(b)]. Also the cis-isomer attaches with its oxygen atoms pointing toward the $\mathrm{Na}$ ions while the azo-group is lifted off the surface [Fig. 4(e)]. Thus, both adsorption geometries differ from the adsorption geometries on metals, on which the azo-group pulls the molecule toward the surface. ${ }^{25}$ The nature of the bonding on $\mathrm{NaCl}$ is visualized by plotting the electrostatic potential of the molecule and of the uncovered $\mathrm{NaCl}$ surface in Figs. 4(a) and 4(d), respectively.

We further analyze the electronic structure of the adsorbed molecules in terms of the projected density of states (pDOS) [Fig. 4(c)]. The pDOS reveals negligible differences in the energetic position and spread of the adsorbed molecule's $\pi, n$, and $\pi^{*}$ orbitals as compared to those of the same isomer in the gas phase. Thus, a suppression of the isomerization induced by visible light is not due to a change in optical transitions. 

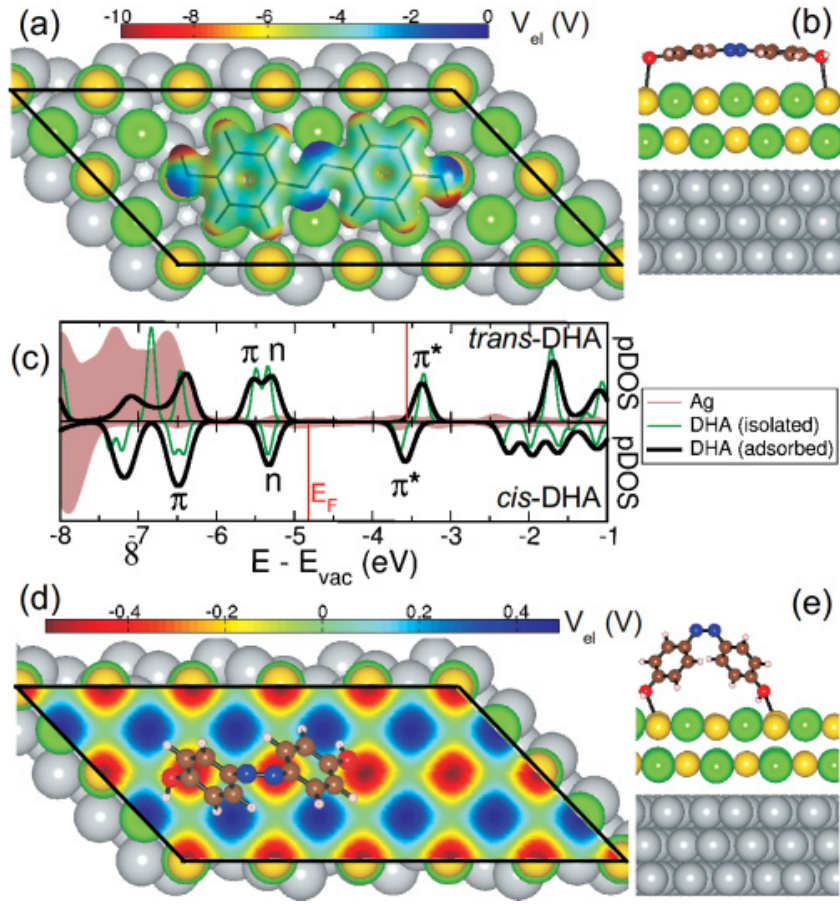

FIG. 4. (Color online) Adsorption geometry of trans-DHA (a), (b) and cis-DHA (d), (e) on $\mathrm{NaCl} / \mathrm{Ag}(111)$. Coloring of atoms: Ag: gray, Cl: green, Na: yellow, C: brown, O: red, N: blue. (a) Top view of trans-DHA with an added electron density isosurface of the molecule color-coded with electrostatic potential. (b) Side view of the trans-DHA adsorption geometry. (c) Partial density of states (pDOS) for trans-DHA (top) and cis-DHA (bottom). The DOS projected on silver atoms (gray) and the DHA molecule (black) is indicated. For comparison, the pDOS of free DHA molecules is added. The Fermi level is shown as a red line, energies are with respect to the vacuum level. (d) Top view of cis-DHA with an added electrostatic potential map of the uncovered $\mathrm{NaCl}$ surface at a distance of $0.28 \mathrm{~nm}$ above the $\mathrm{NaCl}$ surface. (e) Side view of the trans-DHA adsorption geometry.

Particularly, the $n$ orbital, which is localized at the azo-group, remains almost unaffected upon adsorption due to its comparably large distance from the surface. Furthermore, such a minor change also makes a faster deexcitation within the molecule unlikely. Also, the charge-transfer probability to the metal surface across the $0.56-\mathrm{nm}$-thick $\mathrm{NaCl}$ is negligible on the time scale of isomerization. We conclude that electrostic repulsion is the likely cause for suppressing the inversion pathway.

However, why should one ring be hindered to move in parallel to the surface? Here, we should keep in mind the polar nature of the $\mathrm{OH}$ end groups as visualized by the electrostatic isosurface map in Fig. 4(a). In a possible inversion pathway, the $\mathrm{OH}$ group would have to slide over the $\mathrm{Cl}$ anion where electrostatic interactions clearly provide an additional energy barrier, thereby preventing this pathway. This is illustrated in Fig. 5(a). In contrast, the rotation pathway

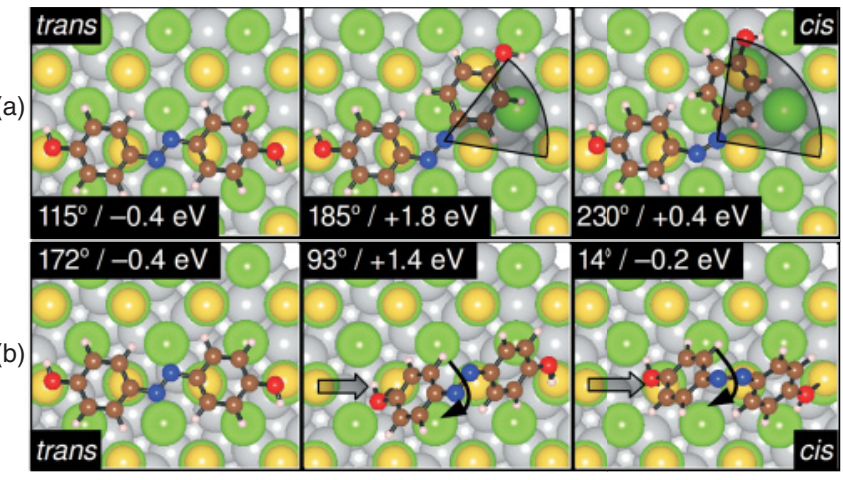

FIG. 5. (Color online) Illustration of two possible isomerization pathways starting from trans-DHA and ending at cis-DHA; top view, atom colors are identical to those of Fig. 4: (a) Snapshots along the inversion pathway; the C-N-N bond angle is indicated in the top left corners of the panels. (b) Snapshots along the rotation pathway; the dihedral angle is indicated.

allows for an energetically more advantageous situation as visualized in Fig. 5(b). One oxygen atom can remain at its favorable adsorption site during the complete isomerization path; the other oxygen atoms slides along a Na column, thereby experiencing only minor electrostatic barriers.

We are aware of the fact that our above arguments are based on the potential energy landscape of adsorbed molecules in their ground state, while a full treatment of the photoisomerization process would require the knowledge of the potential energy surface in an optically excited state. Unfortunately, this is out of reach for molecules adsorbed at surfaces using present-day $a b$ initio methods. Nevertheless, we believe that the ground-state energetics correctly captures the molecule-substrate interactions, which we identify to be responsible for the preference of the rotation over the inversion pathway since the dominant electrostatic interactions of the hydroxy end groups with $\mathrm{Na}$ and $\mathrm{Cl}$ surface ions would be likewise present in optically excited state.

\section{CONCLUSION}

In summary, we have demonstrated a bistable photo switch in contact with a substrate. Switching is feasible via the rotation pathway of trans-cis isomerization but not via the inversion pathway because of electrostatics. This demonstrates that the atomistic details of an inert surface have to be considered because they might significantly influence the nanoscale functionality of single molecules.

\section{ACKNOWLEDGMENTS}

J.H. and K.M. acknowledge financial support from the Deutsche Forschungsgemeinschaft (DFG). P.P and C.A.-D. acknowledge financial support from the Austrian Science Fund (FWF), projects P23190-N16 and S97-14.

\footnotetext{
*Corresponding author: morgenstern@fkp.uni-hannover.de ${ }^{1}$ C. Joachim, J. K. Gimzewski, and A. Aviram, Nature (London) 408, 541 (2000); W. R. Browne and B. L. Feringa, Nature Nanotechn. 1, 25 (2006).
}

${ }^{2}$ N. Tamai and H. Miyasaka, Chem. Rev. 100, 1875 (2000); P. Behrens, A. M. Glaue, and O. Oellrich, in Host-Guest Systems Based on Nanoporous Crystals, edited by F. Laeri, F. Schüth, U. Simon, and M. Wark (Wiley-VCH, Weinheim, 2003), 
pp. 121-144; C.-W. Chang, Y.-C. Lu, T.-T. Wang, and E. W.-G. Diau, J. Am. Chem. Soc. 126, 10109 (2004).

${ }^{3}$ K. Ichimura, S. K. Oh, and M. Nakagawa, Science 288, 1624 (2000); A. S. Kumar, T. Ye, T. Takami, B.-C. Yu, A. K. Flatt, J. M. Tour, and P. S. Weiss, Nano Lett. 8, 1644 (2008).

${ }^{4}$ M. J. Comstock, N. Levy, A. Kirakosian, J. Cho, F. Lauterwasser, J. H. Harvey, D. A. Strubbe, J. M. J. Frechet, D. Trauner, S. G. Louie, and M. F. Crommie, Phys. Rev. Lett. 99, 038301 (2007).

${ }^{5}$ S. Hagen, P. Kate, F. Leyssner, D. Nandi, M. Wolf, and P. Tegeder, J. Chem. Phys. 129, 164102 (2008).

${ }^{6}$ M. J. Comstock, N. Levy, J. Cho, L. Berbil-Bautista, M. F. Crommie, D. A. Poulsen, and J. M. J. Frechet, Appl. Phys. Lett. 92, 123107 (2008).

${ }^{7}$ M. Bazarnik, J. Henzl, R. Czajka, and K. Morgenstern, Chem. Commun. 47, 7764 (2011).

${ }^{8}$ P. Liljeroth, J. Repp, and G. Meyer, Science 317, 1203 (2007).

${ }^{9}$ J. Repp, G. Meyer, F. E. Olsson, and M. Persson, Science 305, 493 (2004).

${ }^{10}$ F. E. Olsson, S. Paavilainen, M. Persson, J. Repp, and G. Meyer, Phys. Rev. Lett. 98, 176803 (2007).

${ }^{11}$ S. Monti, G. Orlandi, and P. Palmieri, Chem. Phys. 71, 87 (1982).

${ }^{12}$ J. P. Perdew, K. Burke, and M. Ernzerhof, Phys. Rev. Lett. 77, 3865 (1996).

${ }^{13}$ S. Grimme, J. Comput. Chem. 27, 1787 (2006).
${ }^{14}$ G. Kresse and J. Furthmüller, Phys. Rev. B 54, 11169 (1996).

${ }^{15}$ J. Henzl, M. Mehlhorn, H. Gawronski, K.-H. Rieder, and K. Morgenstern, Angew. Chem. 118, 617 (2006); Angew. Chem. Int. Ed. 45, 603 (2006); J. Henzl and K. Morgenstern, PhysChemChemPhys 12, 6035 (2010).

${ }^{16}$ B. Y. Choi, S. J. Kahng, S. Kim, H. Kim, H. W. Kim, Y. J. Song, J. Ihm, and Y. Kuk, Phys. Rev. Lett. 96, 156106 (2006).

${ }^{17}$ M. Alemani, M. V. Peters, S. Hecht, K.-H. Rieder, F. Moresco, and L. Grill, J. Am. Chem. Soc. 128, 14446 (2006).

${ }^{18} \mathrm{H}$. Gawronski, J. Henzl, V. Simic-Milosevic, and K. Morgenstern, Appl. Surf. Sci. 253, 9047 (2007).

${ }^{19}$ In addition, some diffusion of single protrusions is induced.

${ }^{20}$ A. Safiei, J. Henzl, and K. Morgenstern, Phys. Rev. Lett. 104, 216102 (2010)

${ }^{21}$ K. Morgenstern, Prog. Surf. Sci. 86, 115 (2011).

${ }^{22}$ The standing time of the liquid-nitrogen reservoir is $30 \mathrm{~h}$; thermalization of the microscope after illumination to $5 \mathrm{~K}$ takes around $3 \mathrm{~h}$; preparation of the tip after filling and after illumination takes approximately $1 \mathrm{~h}$ each; imaging different spots of the surface before and after illumination takes around $3 \mathrm{~h}$ each.

${ }^{23}$ G. Füchsel, T. Klamroth, J. Dokic, and P. Saalfrank, J. Phys. Chem. B 110, 16337 (2006).

${ }^{24}$ M. Wolf and P. Tegeder, Surf. Sci. 603, 1506 (2009).

${ }^{25}$ E. McNellis, J. Meyer, A. D. Baghi, and K. Reuter, Phys. Rev. B 80, 035414 (2009). 